\title{
Vancomycin-resistant Enterococcal Bloodstream Infections in Hematopoietic Stem Cell Transplant Recipients and Patients with Hematologic Malignancies: Impact of Daptomycin MICs of 3 to $4 \mathrm{mg} / \mathrm{L}$
}

Pearlie P. Chong, $\mathrm{MD}^{1}$; David van Duin, $\mathrm{MD}, \mathrm{PhD}^{1}$; Ananta Bangdiwala, MD, $\mathrm{MPH}^{2}$; Anastasia Ivanova, $\mathrm{PhD}^{3}$; William C. Miller, MD, PhD, $\mathrm{MPH}^{4}$; David J. Weber, MD, MPH ${ }^{1,5}$; Peter H. Gilligan, $\mathrm{PhD}^{6}$; and Thomas C. Shea, $\mathrm{MD}^{7}$ ${ }^{1}$ Division of Infectious Diseases, University of North Carolina, Chapel Hill, North Carolina; ${ }^{2}$ Biostatistics and Bioinformatics Core, Masonic Cancer Center, University of Minnesota, Minneapolis, Minnesota; ${ }^{3}$ Department of Biostatistics, University of North Carolina, Chapel Hill, North Carolina; ${ }^{4}$ Division of Epidemiology, College of Public Health, The Ohio State University, Columbus, Ohio; ${ }^{5}$ Hospital Epidemiology, UNC Health Care, Chapel Hill, North Carolina; ${ }^{6}$ Clinical Microbiology-Immunology Laboratories, University of North Carolina Health Care and Department of Pathology-Laboratory Medicine, University of North Carolina School of Medicine, Chapel Hill, North Carolina; and ${ }^{7}$ Division of Hematology/Oncology and UNC Lineberger Comprehensive Cancer Center, Chapel Hill, North Carolina

\begin{abstract}
Purpose: Case reports of treatment failure with standard-dose daptomycin $(6 \mathrm{mg} / \mathrm{kg})$ have recently surfaced in vancomycin-resistant Enterococcus (VRE) bloodstream infection (BSI) episodes with daptomycin MICs of 3 to $4 \mathrm{mg} / \mathrm{L}$. The clinical implications of daptomycin MICs of 3 to $4 \mathrm{mg} / \mathrm{L}$ in VRE BSIs have not been elucidated.
\end{abstract}

Methods: We performed a single institutional retrospective analysis of adult stem cell transplant recipients and patients with hematologic malignancies diagnosed with VRE BSI from 2006 to 2014 and compared outcomes between those with daptomycin MICs of 3 to $4 \mathrm{mg} / \mathrm{L}$ those with $2 \mathrm{mg} / \mathrm{L}$, as determined by Etest.

Findings: Forty-two daptomycin-treated VRE BSI episodes, all due to Enterococcus faecium were identified; 19 episodes with daptomycin MICs of 3 to 4 $\mathrm{mg} / \mathrm{L}$ and 23 episodes with a daptomycin MIC of 2 $\mathrm{mg} / \mathrm{L}$. Patients in the higher daptomycin MIC group were more likely to be male, to be stem cell transplant recipients, and to have received high-dose daptomycin treatment ( $>6 \mathrm{mg} / \mathrm{kg}$ ). In unadjusted analyses, microbiological failure in the daptomycin MICs 3 to $4 \mathrm{mg} / \mathrm{L}$ versus $2 \mathrm{mg} / \mathrm{L}$ groups (odds ratio $=1.79,95 \% \mathrm{CI}$, $0.52-6.11 ; P=0.35)$, the median duration of bacteremia (4 days in daptomycin MICs 3-4 mg/L vs 3 days in daptomycin MIC $2 \mathrm{mg} / \mathrm{L} ; P=0.18$ ) and all-cause 30 -day mortality $(21 \%$ in daptomycin MICs $3-4 \mathrm{mg} / \mathrm{L}$ vs $35 \%$ in daptomycin MIC $2 \mathrm{mg} / \mathrm{L}$ group; $P=0.49)$ were not different. In adjusted analyses, the association between higher Pitt bacteremia scores and all-cause 30-day mortality was statistically significant $(P=0.0006)$, whereas the association between daptomycin MICs of 3 to $4 \mathrm{mg} / \mathrm{L}$ and all-cause 30-day mortality approached statistical significance $(P=0.06)$.

Implications: Duration of bacteremia and microbiological failure rates did not differ by daptomycin MICs in VRE BSI episodes in our patients, composed of adult stem cell transplant recipients and patients with hematologic malignancies. There was a nonsignificant trend in multivariable analysis suggesting that allcause 30-day mortality was lower in patients whose VRE bloodstream isolates were with daptomycin MICs of 3 to $4 \mathrm{mg} / \mathrm{L}$. (Clin Ther. 2016;38:2468-2476) (c) 2016 Elsevier HS Journals, Inc. All rights reserved.

Accepted for publication September 21, 2016 http://dx.doi.org/10.1016/j.clinthera.2016.09.011 
Key words: bacteremia, daptomycin, Enterococcus faecium, neutropenia, vancomycin-resistant enterococci, VRE.

\section{INTRODUCTION}

Vancomycin-resistant enterococcus (VRE) is the most common cause of bloodstream infection (BSI) in allogeneic stem cell transplant (HSCT) recipients at many institutions in the United States. ${ }^{1,2}$ Its cumulative incidence during the first year post-transplantation is estimated to range from $3.6 \%$ to $22 \%$, with 30 -day mortality rates ranging between $38 \%$ and $88 \% .^{1-5}$ Treatment of VRE BSIs can be challenging due to limited number of effective antimicrobial agents. Daptomycin is often used as first-line treatment for its bactericidal activity and is generally well tolerated. According to the Clinical and Laboratory Standards Institute (CLSI), Enterococcus isolates with daptomycin MICs of $\leq 4 \mathrm{mg} / \mathrm{L}$, as determined by broth dilution or Etest, are considered daptomycin susceptible. ${ }^{6}$

Unfortunately, reports of treatment failure using standard-dose daptomycin $(6 \mathrm{mg} / \mathrm{kg})$ monotherapy in BSIs due to Enterococcus faecium with MICs of 3 to $4 \mathrm{mg} / \mathrm{L}$ have recently surfaced, ${ }^{7}$ forcing clinicians to re-evaluate whether the current treatment approach is optimal. The clinical implications and whether a more aggressive treatment strategy should be used in VRE BSI with daptomycin MICs of 3 to $4 \mathrm{mg} / \mathrm{L}$ is currently unknown. In the absence of data from clinical trials to guide management, we performed a retrospective study at our institution to examine whether HSCT recipients and patients with hematologic malignancies in whom VRE BSI develops with daptomycin MICs of 3 to $4 \mathrm{mg} / \mathrm{L}$ have worse outcomes compared with those with an MIC of $2 \mathrm{mg} / \mathrm{L}$.

\section{MATERIALS AND METHODS Study Population}

All patients with $\geq 1$ positive blood cultures for VRE between September 2006 and September 2014 were identified in our computerized microbiology database. This time period was chosen because routine daptomycin susceptibility testing of VRE bloodstream isolates commenced in September 2006 at our institution. A retrospective chart review of adult HSCT recipients and patients with hematologic malignancies (18 years of age and older) with VRE bacteremia treated with daptomycin monotherapy for at least 72 hours was performed. Patients who received VRE-active therapy other than daptomycin were excluded. The University of North Carolina Institutional Review Board approved this study.

\section{Definitions}

VRE BSI was defined as the isolation of vancomycin-resistant Enterococcus species from $\geq 1$ blood cultures. Duration of bacteremia was defined as the number of days between the first positive and first negative blood cultures. VRE BSIs were considered recurrent if VRE was isolated from blood cultures after completion of appropriate antibiotic treatment course with $\geq 1$ negative interim blood cultures and resolution of symptoms associated with the first BSI episode. A copathogen was defined as any pathogen other than VRE isolated from $\geq 1$ blood cultures within the same BSI episode. Daptomycin susceptibility was defined as an MIC of $\leq 4 \mathrm{mg} / \mathrm{L}$ according to the CLSI criteria and determined using Etest for the entire study period. VRE isolates with a daptomycin MIC of $3 \mathrm{mg} / \mathrm{L}$ were rounded up to the next doubling dilution $(4 \mathrm{mg} / \mathrm{L}$ ) and were reported as such. Effective antibiotic therapy was defined as the antibiotic(s) with activity against VRE with which negative blood cultures was attained.

We used the Pitt bacteremia score to measure severity of illness in this study. The maximum score 48 hours before or on the day of the first positive blood culture was recorded and considered the Pitt bacteremia score for that VRE BSI episode. The severity of VRE BSI episodes was also assessed by the presence or absence of septic shock, defined as hypotension requiring any amount of vasopressor(s). The source of VRE BSI was categorized as central line-associated bloodstream infection, gastrointestinal or other sites such as the urinary tract, osteomyelitis, and cellulitis. BSIs occurring in the presence of a central venous catheter or within 48 hours of removal of a central venous catheter and that cannot be attributed to an infection unrelated to the catheter were considered to be central line-associated bloodstream infections, as defined by the National Healthcare Safety Network. ${ }^{8}$ The presence of radiographic or endoscopic evidence of enterocolitis within 7 days of the first positive blood culture for VRE was considered gastrointestinal in origin.

Primary outcomes of interest include the duration of bacteremia for VRE BSIs, all-cause 30-day 
mortality, and microbiological failure. Microbiological failure was defined as clearance occurring $\geq 4$ days after the index blood culture that included at least 72 hours of daptomycin therapy or if the patient died with persistently positive cultures. ${ }^{\text {? }}$

\section{Data Abstraction}

Data abstracted from electronic medical records include age at the time of bacteremia, sex, race, dates of onset and resolution of neutropenia, administration of chemotherapy, history of bone marrow transplantation, VRE colonization status before diagnosis of the first BSI episode, dates of all positive and negative blood and catheter tip cultures, urine cultures, radiographic or endoscopic evidence of biliary or colorectal pathology within 7 days of the first positive blood culture for VRE, MICs of antibiotics with activity against Enterococcus spp, details of daptomycin treatment at the time of VRE BSI diagnosis including the initial dose and duration, Pitt bacteremia scores, the presence of septic shock and need for intensive care unit admission, length of hospital stay, and date and cause of death.

\section{Protocols}

All patients are screened for VRE colonization on admission to the bone marrow transplantation unit at our institution if $\geq 30$ days has lapsed since the last stool culture was performed. Weekly VRE screening is then performed on all patients in the bone marrow transplantation unit with negative initial VRE screening results, and those found to be colonized or infected with VRE were managed using under contact precautions. Stool or rectal swabs were inoculated onto VRE chromogenic agar (bioMérieux, Marcy l'Étoile, France). Plates were incubated at $35^{\circ} \mathrm{C}$ and read at 16 to 24 hours and again at 36 to 48 hours for the presence of distinctive purple or blue colonies. Isolates identified as E faecium or fecalis by MALDI-TOF mass spectroscopy (bioMerieux, Durham, NC) were plated on Brain Heart Infusion Agar containing $6 \mu \mathrm{g} / \mathrm{mL}$ vancomycin to confirm vancomycin resistance.

Patients with neutropenia who were afebrile received levofloxacin prophylaxis, and neutropenia was defined by an absolute neutrophil count $\leq 500 / \mathrm{mm}^{3}$. Initial and repeat blood cultures were drawn from central venous catheter(s) or peripheral sites with new onset of fever or with other signs of infection. Daptomycin dose determination was based on patients' actual body weight, with high-dose daptomycin defined as $>6 \mathrm{mg} / \mathrm{kg}$. Frequencies of repeat blood cultures after initiation of treatment were at the discretion of the treating physician.

\section{Statistical Analysis}

Only the first VRE BSI episodes per patient with daptomycin MICs of 3 to 4 or $2 \mathrm{mg} / \mathrm{L}$ were analyzed. Comparisons of BSI episodes with daptomycin MICs of 3 to 4 and $2 \mathrm{mg} / \mathrm{L}$ were made using a $\chi^{2}$ test, a 2 -sided Fisher exact test, or a Wilcoxon test where appropriate. Time-to-event analysis was performed using the Kaplan-Meier method, with death being the event of interest. Censoring was performed for patients who were alive at day 30 from the date of the first positive blood culture (for 30-day mortality). All relevant clinical and laboratory variables were first tested by univariable Cox models. Variables with $P<0.20$ were then included in the multivariable Cox model to identify potential risk factors for all-cause 30-day mortality. Logistic regression modeling was performed to evaluate risk factors for microbiological failure. A sensitivity analysis was performed to assess the impact of high-dose daptomycin treatment on 30-day mortality by imputation for missing values. All statistical analyses in this study were performed using SAS statistical software (version 9.4, SAS Institute Inc., Cary, NC).

\section{RESULTS \\ VRE Episodes and Patient Characteristics}

A total of 72 VRE BSI episodes were identified in 59 adult HSCT recipients and patients with hematologic malignancies at our institution during this 8-year study period. Forty-two first VRE BSI episodes met criteria to be included in this study (Figure 1). Seventeen patients with VRE BSI were excluded for the following reasons: daptomycin MIC not available $(\mathrm{n}=1)$, daptomycin $\mathrm{MIC}<2 \mathrm{mg} / \mathrm{L} \quad(\mathrm{n}=1)$, daptomycin $\mathrm{MIC}>4 \mathrm{mg} / \mathrm{L}(\mathrm{n}=5)$, inadequate follow-up information due to transfer of care to a different institution $(\mathrm{n}=2)$, and use of combination therapy or antibiotic therapy other than daptomycin $(\mathrm{n}=8)$. In these 42 VRE BSI episodes, all were due to E faecium; $19(45 \%)$ and $23(55 \%)$ isolates had daptomycin MICs of 3 to $4 \mathrm{mg} / \mathrm{L}$ and $2 \mathrm{mg} / \mathrm{L}$, respectively (Figure 1). 


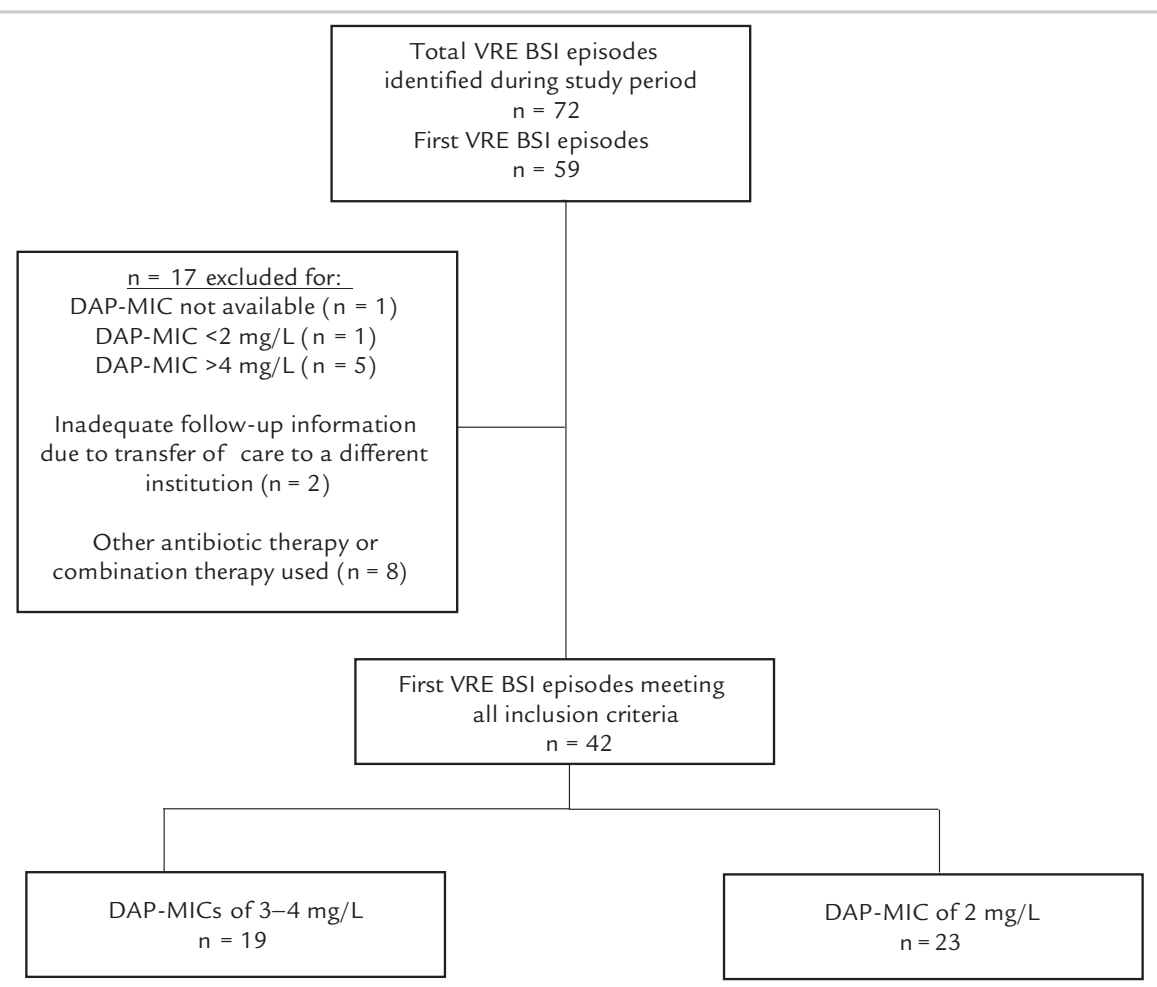

Figure 1. Flowchart for the primary analysis of VRE BSI episodes. BSI = bloodstream infection; DAP = daptomycin; VRE = vancomycin-resistant Enterococcus.

Patient and transplant characteristics stratified by daptomycin MIC are shown in Table I. Sex, transplantation status, and high-dose daptomycin use

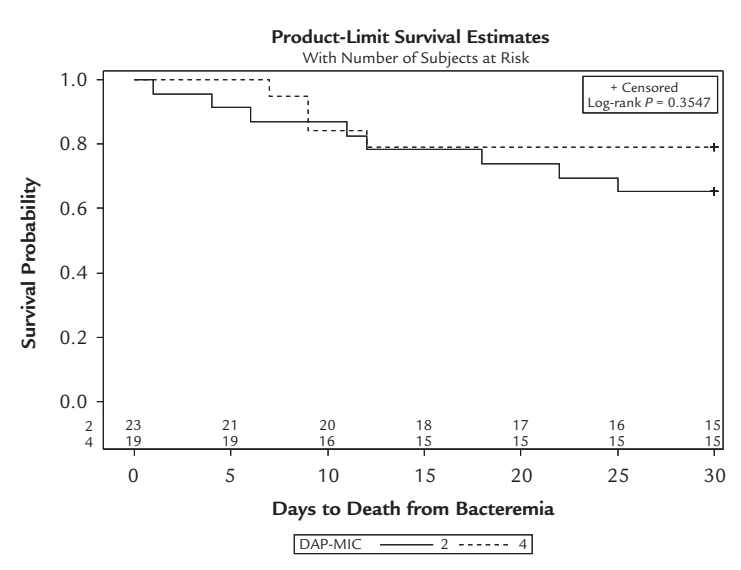

Figure 2. Survival probabilities on day 30 from date of first positive blood culture for VRE, as estimated by the Kaplan-Meier method. VRE = vancomycin-resistant Enterococcus. were significantly different between the 2 groups (Table I). Patients with VRE BSI episodes with daptomycin MICs of 3 to $4 \mathrm{mg} / \mathrm{L}$ were less likely to be female $(26 \%$ vs $57 \%, P=0.049)$, were more likely to be HSCT recipients $(74 \%$ vs $30 \%, P=0.006)$, and were more likely to be treated with high-dose daptomycin $(71 \%$ [10 of 14 ] vs $16 \%$ [ 4 of 19 ], $P=0.001)$. Other characteristics such as duration of neutropenia, Pitt bacteremia scores, intensive care unit stay, source of VRE BSI, and septic shock were comparable between the 2 groups.

Copathogens were identified in 8 of 42 episodes $(19 \%)$, the most common of which was coagulasenegative Staphylococcus ( $\mathrm{n}=4)$. Other copathogens include Candida glabrata $(\mathrm{n}=2)$, Leuconostoc $(\mathrm{n}=$ 1), and Escherichia coli and Klebsiella spp $(\mathrm{n}=1)$. Central venous catheters were the most common source of infection (22 of 42 [52\%]), followed by gastrointestinal tract (8 of $42[19 \%]$ ), and other (biliary, 1 of 42 [2.5\%], osteomyelitis, 1 of 42 [2.5\%], and unknown, 10 of 42 [24\%]). Recurrent VRE BSIs occurred in 7 of 42 episodes (17\%): 2 in the daptomycin MICs 3 to $4 \mathrm{mg} / \mathrm{L}$ group and 5 in the daptomycin MIC $2 \mathrm{mg} / \mathrm{L}$ group. 
Table I. Patient and transplantation characteristics.

\begin{tabular}{|c|c|c|c|c|}
\hline Characteristics & $\begin{array}{l}\text { DAP MIC 3-4 } \\
\quad(n=19)\end{array}$ & $\begin{array}{l}\text { DAP MIC } 2 \\
(n=23)\end{array}$ & $\begin{array}{l}\text { All Episodes } \\
\qquad(\mathrm{N}=42)\end{array}$ & $P$ \\
\hline Age, $y^{*}, \dagger$ & $54(42-64)$ & $52(41-58)$ & $53(41-62)$ & 0.43 \\
\hline Female sex & $5(26.3)$ & $13(56.5)$ & $18(42.9)$ & 0.049 \\
\hline $\operatorname{Race}^{\ddagger}$ & & & & 1 \\
\hline White & $13(68.4)$ & $15(65.2)$ & $28(66.7)$ & \\
\hline African-American & $4(21.1)$ & $5(21.7)$ & $9(21.4)$ & \\
\hline Other & $2(10.5)$ & $3(13.0)$ & $5(11.9)$ & \\
\hline Primary diagnosis & & & & 0.67 \\
\hline ALL & $2(10.5)$ & $4(17.4)$ & $6(14.3)$ & \\
\hline AML & $11(57.9)$ & $12(52.2)$ & $23(54.8)$ & \\
\hline CLL & $0(0.0)$ & $2(8.7)$ & $2(4.8)$ & \\
\hline CML & $1(5.3)$ & $2(8.7)$ & $3(7.1)$ & \\
\hline Other & $5(26.3)$ & $3(13.0)$ & $8(19.0)$ & \\
\hline HSCT recipient & & & & 0.006 \\
\hline Autologous & $3(15.8)$ & $0(0.0)$ & $3(7.1)$ & \\
\hline Allogeneic & $11(57.9)$ & $7(30.4)$ & $18(42.9)$ & \\
\hline None & $5(26.3)$ & $16(69.6)$ & $21(50.0)$ & \\
\hline Preparative regimen & & & & 1 \\
\hline Myeloablative & $9(42.1)$ & $4(17.4)$ & $13(28.6)$ & \\
\hline RIC & $5(26.3)$ & $3(13.0)$ & $8(19.0)$ & \\
\hline Stem-cell source & & & & 1 \\
\hline Bone marrow & $1(5.3)$ & $1(4.3)$ & $2(4.8)$ & \\
\hline Peripheral & $10(52.6)$ & $6(26.1)$ & $16(38.1)$ & \\
\hline Transplant donor type & & & & 0.64 \\
\hline Unrelated & $6(31.6)$ & $5(21.7)$ & $11(26.2)$ & \\
\hline Related & $5(26.3)$ & $2(8.7)$ & $7(16.7)$ & \\
\hline Duration of neutropenia, days ${ }^{*}, \dagger$ & $11(0-19)$ & $16(10-22)$ & $13.5(3-19)$ & 0.39 \\
\hline Pitt bacteremia score & $1(0-4)$ & $1(0-3)$ & $1(0-3)$ & 0.14 \\
\hline ICU stay & $6(31.6)$ & $8(34.8)$ & $14(33.3)$ & 0.83 \\
\hline Septic shock & $6(31.6)$ & $5(21.7)$ & $11(26.2)$ & 0.50 \\
\hline Source of BSI & & & & 0.27 \\
\hline Catheter related & $8(42.1)$ & $14(60.9)$ & $22(52.4)$ & \\
\hline Gastrointestinal & $3(15.8)$ & $5(21.7)$ & $8(19.1)$ & \\
\hline Other & $8(42.1)$ & $4(17.4)$ & $12(29.6)$ & \\
\hline VRE colonized ${ }^{\S}$ & $12(63.2)$ & $9(39.1)$ & $21(50)$ & 0.12 \\
\hline High-dose daptomycin treatment ${ }^{\|}$ & $10 / 14(71.4)$ & $4 / 19(15.8)$ & $13(39.4)$ & 0.001 \\
\hline Daptomycin treatment duration, days ${ }^{*}$ & $15(10-26)$ & $14(12-21)$ & $15(12-22)$ & 0.42 \\
\hline
\end{tabular}

$\mathrm{ALL}=$ acute lymphoblastic leukemia; $\mathrm{AML}=$ acute myeloid leukemia; $\mathrm{BSI}=$ bloodstream infection; $\mathrm{CLL}=$ chronic lymphocytic leukemia; CML = chronic myeloid leukemia; DAP = daptomycin; HSCT = hematopoietic stem cell transplant; $\mathrm{ICU}=$ intensive care unit; RIC = reduced intensity conditioning; VRE = vancomycin-resistant enterococcus.

*Values are median (interquartile ranges).

${ }^{\dagger}$ At the time of bloodstream infection diagnosis.

${ }^{\ddagger}$ Values are number (\%).

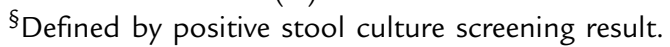

"Defined as $>6 \mathrm{mg} / \mathrm{kg}$ of actual body weight. 
Table II. Risk factors for all-cause 30-day mortality among patients with VRE BSI using Cox models.

\begin{tabular}{|c|c|c|c|c|c|c|}
\hline Variable & Hazard Ratio & $95 \% \mathrm{Cl}$ & $P$ & $\begin{array}{c}\text { Adjusted } \\
\text { Hazard Ratio }\end{array}$ & $95 \% \mathrm{Cl}$ & $P$ \\
\hline $\mathrm{Age}^{*}$ & 1.02 & $0.98-1.06$ & 0.359 & & & \\
\hline Male sex & 0.75 & $0.24-2.32$ & 0.615 & & & \\
\hline $\mathrm{AML}$ as primary disease & 0.36 & $0.11-1.21$ & 0.099 & 0.41 & $0.12-1.39$ & 0.154 \\
\hline HSCT recipient & 1.48 & $0.47-4.67$ & 0.502 & & & \\
\hline RIC regimen & 0.38 & $0.04-3.39$ & 0.378 & & & \\
\hline Duration of neutropenia* $^{*}$ & 0.98 & $0.94-1.02$ & 0.352 & & & \\
\hline VRE colonized $^{\dagger}$ & 1.45 & $0.46-4.57$ & 0.525 & & & \\
\hline Pitt bacteremia score ${ }^{*}$ & 1.49 & $1.17-1.89$ & 0.001 & 1.65 & $1.24-2.19$ & 0.0006 \\
\hline Daptomycin MIC 3-4 mg/L $\mathrm{L}^{\ddagger}$ & 0.57 & $0.17-1.90$ & 0.363 & 0.27 & $0.07-1.06$ & 0.060 \\
\hline High-dose daptomycin treatment ${ }^{\S}$ & 0.62 & $0.12-3.06$ & 0.55 & & & \\
\hline
\end{tabular}

$\mathrm{AML}=$ acute myeloid leukemia; $\mathrm{BSI}=$ bloodstream infection; $\mathrm{HSCT}=$ hematopoietic stem cell transplant; RIC = reducedintensity conditioning; VRE = vancomycin-resistant enterococcus.

${ }^{*}$ Analyzed as a continuous variable.

${ }^{\dagger}$ Defined by positive stool culture screening results.

${ }^{\ddagger}$ Reference group: daptomycin MIC $=2 \mathrm{mg} / \mathrm{L}$.

$\S$ Only performed in patients for whom daptomycin treatment dose $(n=33)$ was known.

\section{Outcomes of Patients With VRE BSI}

Survival probabilities on day 30 from date of first positive blood culture for VRE were not different between the daptomycin MICs of 3 to $4 \mathrm{mg} / \mathrm{L}$ group and $2 \mathrm{mg} / \mathrm{L}$ group $(79 \%$ vs. $65 \% ; p=0.355)$ (Figure 2), as estimated by the Kaplan-Meier method. Results of unadjusted and adjusted Cox models examining the relationships between possible risk factors and all-cause 30-day mortality from the time of VRE BSI diagnosis are shown in Table II. In an unadjusted analysis, the hazard ratio of all-cause 30-day mortality was 0.57 in the daptomycin MICs of 3 to $4 \mathrm{mg} / \mathrm{L}$ group compared with the daptomycin MIC of $2 \mathrm{mg} / \mathrm{L}$ group $(95 \% \mathrm{CI}, 0.17-1.90 ; P=$ 0.363). After adjusting for Pitt bacteremia score and acute myeloid leukemia as the primary diagnosis, this association approached statistical significance $(P=$ 0.06) (Table II). A higher Pitt bacteremia score was associated with increased 30-day mortality in both unadjusted and adjusted analysis (adjusted hazard ratio $=1.65 ; 95 \% \mathrm{CI}, 1.24-2.19 ; P=0.0006$ ). None of the risk factors including higher daptomycin MICs was associated with microbiological failure in logistic regression modeling (Table III). Using deathcensored time to event analysis, the median duration of bacteremia was 4 days (interquartile range [IQR],
2-6 days) versus 3 days (IQR, 2-4 days) in the daptomycin MICs $3-4 \mathrm{mg} / \mathrm{L}$ and $2 \mathrm{mg} / \mathrm{L}$ groups, respectively. This difference was not statistically significant by log-rank test $(P=0.184)$.

\section{Treatment}

All VRE BSI episodes were treated with daptomycin. The median duration of daptomycin treatment was 15 days (IQR, 10-26) versus 14 days (IQR, 12-21) $(P=0.42)$ in the daptomycin MIC 3 to $4 \mathrm{mg} / \mathrm{L}$ and daptomycin MIC $2 \mathrm{mg} / \mathrm{L}$ groups, respectively. However, information on daptomycin dose was unavailable in 9 BSI episodes $(21 \%)$. Thirteen of the 33 episodes $(39 \%)$ were treated with initial high-dose daptomycin $(>6 \mathrm{mg} / \mathrm{kg})$ with a median daptomycin dose of $8.1 \mathrm{mg} / \mathrm{kg}$ (IQR, 7-10 mg/kg). VRE BSI episodes with daptomycin MICs of 3 to $4 \mathrm{mg} / \mathrm{L}$ were significantly more likely to have received high-dose treatment compared with BSI episodes with daptomycin MIC $2 \mathrm{mg} / \mathrm{L}$ (10 of 14 [71\%] vs 4 of 19 [16\%]; $P=0.001$ ) (Table I). Imputing for the 9 missing daptomycin dose values using sensitivity analysis (data not shown), the mean adjusted HR for 30-day mortality for the daptomycin MICs 3 to $4 \mathrm{mg} / \mathrm{L}$ group was 0.15 (adjusted HR range, 0.05- 0.29), adjusting for high-dose daptomycin use and Pitt bacteremia score. 


\begin{tabular}{|c|c|c|c|}
\hline & Odds & & \\
\hline Variable & Ratio & $95 \% \mathrm{Cl}$ & $P$ \\
\hline $\begin{array}{l}\text { Daptomycin MIC } \\
3-4 \mathrm{mg} / \mathrm{L}^{*}\end{array}$ & 1.79 & $0.52-6.11$ & 0.354 \\
\hline $\begin{array}{l}\text { Duration of } \\
\text { neutropenia }^{\dagger}\end{array}$ & 0.97 & $0.94-1.01$ & 0.154 \\
\hline VRE colonized $^{\ddagger}$ & 2.64 & $0.76-9.18$ & 0.127 \\
\hline Pitt bacteremia score ${ }^{\dagger}$ & 0.88 & $0.64-1.21$ & 0.435 \\
\hline HSCT recipient & 2.64 & $0.76-9.18$ & 0.127 \\
\hline $\begin{array}{l}\text { Central Line as source } \\
\text { of BSI }\end{array}$ & 1.47 & $0.43-4.95$ & 0.537 \\
\hline $\begin{array}{l}\text { High-dose daptomycin } \\
\text { treatment }^{\S}\end{array}$ & 1.17 & $0.29-4.23$ & 0.829 \\
\hline
\end{tabular}

$\mathrm{BSI}=$ bloodstream infection; HSCT $=$ hematopoietic stem cell transplant; MIC = minimum inhibitory concentration; VRE, vancomycin-resistant enterococcus. ${ }^{*}$ Reference group: daptomycin MIC $=2$.

${ }^{\dagger}$ Analyzed as a continuous variable.

${ }^{\ddagger}$ Defined by positive stool culture screening result.

$\S$ Only performed in patients in whom daptomycin treatment dose $(n=33)$ was known.

None of these patients experienced adverse events attributed to high-dose daptomycin use.

\section{DISCUSSION}

To the best of our knowledge, this is the first study to demonstrate that the duration of bacteremia and microbiological failure rates did not differ in HSCT recipients and patients with hematologic malignancies in whom VRE BSIs develop with daptomycin MICs of 3 to $4 \mathrm{mg} / \mathrm{L}$ compared with a daptomycin MIC of $2 \mathrm{mg} / \mathrm{L}$. Interestingly, after adjusting for the Pitt bacteremia score and acute myeloid leukemia as the primary disease, the 30-day mortality rate was lower in the group with higher daptomycin MICs, and this approached statistical significance, although the unadjusted 30-day mortality rates did not differ between the 2 groups.

VRE BSI is a common infectious complication associated with a high mortality rate in HSCT recipients and patients with hematologic malignancies. ${ }^{1,3,4}$
Treatment options for this multidrug-resistant grampositive infection are limited. Daptomycin is often used as first-line therapy for its bactericidal activity against VRE and lack of marrow toxicity. The optimal daptomycin dose for the treatment of enterococcal infections has yet to be established, although $6 \mathrm{mg} / \mathrm{kg} /$ day, the dose approved by the US Food and Drug Administration for the treatment of Staphylococcus aureus bacteremia, is commonly used. ${ }^{10,11}$ Data from randomized, controlled trials are currently lacking, and whether all patients with VRE BSI should receive high-dose daptomycin remains controversial. ${ }^{7,12-15}$

Because of genomic analyses demonstrating the presence of LiaRS mutations in E faecium bloodstream isolates with daptomycin MICs of $\geq 3 \mathrm{mg} / \mathrm{L}^{16-19}$ and a case report of high-dose daptomycin $(8 \mathrm{mg} / \mathrm{kg} / \mathrm{d})$ failing in daptomycin-sensitive VRE bacteremia (MIC 3 $\mathrm{mg} / \mathrm{L}),{ }^{20}$ the CLSI daptomycin susceptibility breakpoint of $\leq 4 \mathrm{mg} / \mathrm{L}$ for Enterococcus spp has recently been called into question. Substitutions in LiaFSR (a regulatory system that orchestrates the cell envelope stress response in gram-positive bacteria) have been implicated as one of the major pathways in daptomycin resistance. $^{16,18}$ These studies suggest that $E$ faecium isolates with daptomycin MIC values close to $4 \mathrm{mg} / \mathrm{L}$ could harbor resistance mutations, potentially rendering daptomycin ineffective. Because performing gene sequencing on every clinical VRE isolate is not feasible at this time and until a decision is made to lower the daptomycin susceptibility breakpoint by CLSI, we decided to evaluate and report our single-center experience by comparing outcomes in VRE BSI with daptomycin MICs of 3 to $4 \mathrm{mg} / \mathrm{L}$ versus $2 \mathrm{mg} / \mathrm{L}$.

A recent multicenter retrospective study by Shukla et $\mathrm{al}^{9}$ demonstrated that the odds of microbiological failure in E faecium bacteremias with higher daptomycin MICs $(3-4 \mathrm{mg} / \mathrm{L})$ was 4.7 times that of daptomycin MICs of $\leq 2 \mathrm{mg} / \mathrm{L}$, although the in-hospital mortality rates were not different. ${ }^{9}$ Although the likelihood of microbiological failure was also higher in the group with daptomycin MICs of 3 of $4 \mathrm{mg} / \mathrm{L}$, the magnitude of this association was smaller and not statistically significant in our study. This could be due to differences in the 2 study populations. Our study included only patients with hematologic malignancies and/or HSCT recipients, whereas Shukla et $\mathrm{al}^{9}$ included all patients with VRE BSI regardless of underlying diagnosis. Confirmation by future studies in a patient population similar to ours would be helpful. 
We observed that a higher Pitt bacteremia score was significantly associated with higher 30-day mortality rates, even after adjusting for acute myeloid leukemia as the primary diagnosis and higher daptomycin MICs. Although the Charlson Comorbidity Index is widely used in clinical research, its accuracy in retrospective studies such as ours may be affected by poor documentation given the multitude of items required to calculate a score. On the other hand, the components of the Pitt bacteremia score may be more readily abstracted. ${ }^{20}$ It was previously validated as a tool to predict both shortand long-term mortality in patients with gram-negative BSI $^{21-24}$ and methicillin-resistant $S$ aureus BSI. ${ }^{25,26}$ Our study and 1 other retrospective cohort study ${ }^{27}$ suggest that the Pitt bacteremia score may be a reasonable alternative to the Charlson Comorbidity Index as a severity of illness score in enterococcal BSIs.

The limitations of our study include its retrospective study design and small sample size. The frequencies by which repeat blood cultures were drawn to document clearance were not standardized. Daptomycin MICs were determined using Etest and were not confirmed by the broth microdilution method or repeat testing. The initial daptomycin dose was unavailable in some of episodes due to transition of pharmacy software during the study period, and the dose received by these patients was not recorded by physicians in electronic medical records. We were unable to analyze high-dose daptomycin therapy as a covariate in our multivariable model due to the small number of patients. We also did not include time from collection of the first positive blood culture to initiation of daptomycin in our analyses. Our study was a single-center, observational investigation that incorporated adult HSCT recipients and those with underlying hematologic malignancies, thus limiting its generalizability to other patient populations.

In conclusion, we did not observe a significant difference in the duration of bacteremia or microbiological failure rates between patients in whom VRE BSIs developed with daptomycin MICs of 3 to $4 \mathrm{mg} / \mathrm{L}$ versus $2 \mathrm{mg} / \mathrm{L}$. We found a lower 30-day mortality rate for patients in the daptomycin MICs of 3 to $4 \mathrm{mg} / \mathrm{L}$ group. Whether this can be attributed to high-dose daptomycin treatment deserves further exploration. However, several limitations, most notably the small sample size, could have influenced our results. We hope that adequately powered prospective studies will be performed in the future to confirm our study findings.

\section{ACKNOWLEDGMENTS}

We gratefully acknowledge Alan Kerr of McLendon Clinical Laboratories at UNC Hospitals for his assistance with the microbiology database. This research did not receive any specific grant from funding agencies in the public, commercial, or not-for-profit sectors.

\section{CONFLICTS OF INTEREST}

Dr. van Duin has received compensation as a member of the Scientific Advisory Board of Sanofi-Pasteur, Tetraphase, Astellas, and Actavis. Dr. Shea has received compensation as a member of the Scientific Advisory Boards of Spectrum Pharmaceuticals, Otsuka, Bristol-Myers Squibb, Novartis, Millennium, and GlaxoSmithKline. Dr. Chong, Ms. Bangdiwala, Dr. Ivanova, Dr. Miller, Dr. Weber, and Dr. Gilligan declare no potential conflicts of interest.

\section{REFERENCES}

1. Avery R, Kalaycio M, Pohlman B, et al. Early vancomycinresistant enterococcus (VRE) bacteremia after allogeneic bone marrow transplantation is associated with a rapidly deteriorating clinical course. Bone Marrow Transplant. 2005;35:497-499.

2. Kamboj M, Chung D, Seo SK, et al. The changing epidemiology of vancomycin-resistant Enterococcus (VRE) bacteremia in allogeneic hematopoietic stem cell transplant (HSCT) recipients. Biol Blood Marrow Transplant. 2010;16:1576-1581.

3. Weinstock DM, Conlon M, lovino C, et al. Colonization, bloodstream infection, and mortality caused by vancomycin-resistant enterococcus early after allogeneic hematopoietic stem cell transplant. Biol Blood Marrow Transplant. 2007;13:615-621.

4. Vydra J, Shanley RM, George I, et al. Enterococcal bacteremia is associated with increased risk of mortality in recipients of allogeneic hematopoietic stem cell transplantation. Clin Infect Dis. 2012;55:764-770.

5. Satlin MJ, Soave R, Racanelli AC, et al. The emergence of vancomycin-resistant enterococcal bacteremia in hematopoietic stem cell transplant recipients. Leuk Lymphoma. 2014;55:2858-2865.

6. Clinical and Laboratory Standards Institute. Performance Standards for Antimicrobial Susceptibility Testing; Twenty-Fourth Informational Supplement. CLSI document M100-S24. 2014:34. 
7. King EA, McCoy D, Desai S, et al. Vancomycin-resistant enterococcal bacteraemia and daptomycin: are higher doses necessary? J Antimicrob Chemother. 2011;66:2112-2118.

8. Horan TC, Andrus M, Dudeck MA. CDC/NHSN surveillance definition of health care-associated infection and criteria for specific types of infections in the acute care setting. Am J Infect Control. 2008;36:309-332.

9. Shukla BS, Shelburne S, Reyes K, et al. Influence of Minimum Inhibitory Concentration in Clinical Outcomes of Enterococcus faecium Bacteremia Treated With Daptomycin: Is it Time to Change the Breakpoint? Clin Infect Dis. 2016;62:1514-1520.

10. CUBICIN (package insert). Lexington, MA: Cubist Pharmaceuticals, Inc; 2008.

11. Safdar N, Andes D, Craig WA. In vivo pharmacodynamic activity of daptomycin. Antimicrob Agents Chemother. 2004;48:63-68.

12. Kullar R, Davis SL, Levine DP, et al. High-dose daptomycin for treatment of complicated gram-positive infections: a large, multicenter, retrospective study. Pharmacotherapy. 2011;31:527-536.

13. Casapao AM, Kullar R, Davis SL, et al. Multicenter study of high-dose daptomycin for treatment of enterococcal infections. Antimicrob Agents Chemother. 2013;57:4190-4196.

14. Moise PA, Hershberger E, AmodioGroton MI, Lamp KC. Safety and clinical outcomes when utilizing high-dose ( $>$ or $=8 \mathrm{mg} / \mathrm{kg}$ ) daptomycin therapy. Ann Pharmacother. 2009;43:1211-1219.

15. Munita JM, Panesso D, Diaz L, et al. Correlation between mutations in liaFSR of Enterococcus faecium and MIC of daptomycin: revisiting daptomycin breakpoints. Antimicrob Agents Chemother. 2012;56:4354-4359.

16. Tran TT, Panesso D, Gao H, et al. Whole-genome analysis of a daptomycin-susceptible enterococcus faecium strain and its daptomycin-resistant variant arising during therapy. Antimicrob Agents Chemother. 2013;57:261-268.

17. Arias CA, Panesso D, McGrath DM, et al. Genetic basis for in vivo daptomycin resistance in enterococci. $N$ Engl J Med. 2011;365:892-900.

18. Diaz L, Tran TT, Munita JM, et al. Whole-genome analyses of Enterococcus faecium isolates with diverse daptomycin MICs. Antimicrob Agents Chemother. 2014;58:4527-4534.

19. Munita JM, Mishra NN, Alvarez D, et al. Failure of high-dose daptomycin for bacteremia caused by daptomycinsusceptible Enterococcus faecium harboring LiaSR substitutions. Clin Infect Dis. 2014;59:1277-1280.

20. Chow JW, Fine MJ, Shlaes DM, et al. Enterobacter bacteremia: clinical features and emergence of antibiotic resistance during therapy. Ann Intern Med. 1991;115:585-590.

21. Al-Hasan MN, Juhn YJ, Bang DW, et al. External validation of bloodstream infection mortality risk score in a population-based cohort. Clin Microbiol Infect. 2014;20:886-891.

22. Chang TY, Lee CH, Liu JW. Clinical characteristics and risk factors for fatality in patients with bloodstream infections caused by glucose non-fermenting gram-negative Bacilli. J Microbiol Immunol Infect. 2010;43:233-239.
23. Kang Cl, Chung DR, Ko KS, et al. Risk factors for infection and treatment outcome of extended-spectrum betalactamase-producing Escherichia coli and Klebsiella pneumoniae bacteremia in patients with hematologic malignancy. Ann Hematol. 2012;91: 115-121.

24. Paterson DL, Ko WC, Von Gottberg A, et al. International prospective study of Klebsiella pneumoniae bacteremia: implications of extended-spectrum betalactamase production in nosocomial Infections. Ann Intern Med. 2004;140: 26-32.

25. Gasch O, Camoez M, Dominguez $M A$, et al. Predictive factors for early mortality among patients with methicillin-resistant Staphylococcus aureus bacteraemia. J Antimicrob Chemother. 2013;68:1423-1430.

26. Retamar P, Portillo MM, LopezPrieto MD, et al. Impact of inadequate empirical therapy on the mortality of patients with bloodstream infections: a propensity score-based analysis. Antimicrob Agents Chemother. 2012;56:472-478.

27. Cheah AL, Spelman T, Liew D, et al. Enterococcal bacteraemia: factors influencing mortality, length of stay and costs of hospitalization. Clin Microbiol Infect. 2013;19: E181-E189.
Address correspondence to: Pearlie P. Chong, MD, Division of Infectious
Diseases, UNC Chapel Hill, 130 Mason Farm Road, Suite 2134, Campus Box
7030, Chapel Hill, NC 27599-7030. E-mail: pearlie_chong@med.unc.edu 